\title{
Solid supported multicomponent lipid membranes studied by $x$-ray spectromicroscopy
}

\author{
Eva Nováková and Genoveva Mitrea \\ Institut für Röntgenphysik, Universität Göttingen, Friedrich-Hund-Platz 1, 37077 Göttingen, Germany \\ Christian Peth \\ Laser-Laboratorium Göttingen, e.V. Hans-Adolf-Krebs-Weg 1, 37077 Göttingen, Germany \\ Jürgen Thieme \\ Institut für Röntgenphysik, Universität Göttingen, Friedrich-Hund-Platz 1, 37077 Göttingen, Germany \\ Klaus Mann \\ Laser-Laboratorium Göttingen, e.V. Hans-Adolf-Krebs-Weg 1, 37077 Göttingen, Germany \\ Tim Salditt ${ }^{\mathrm{a})}$ \\ Institut für Röntgenphysik, Universität Göttingen, Friedrich-Hund-Platz, 1, 37077 Göttingen, Germany
}

(Received 2 May 2008; accepted 1 August 2008; published 17 September 2008)

\begin{abstract}
This article addresses the lateral organization of two-component lipid membranes deposited on a solid support with the addition of colloidal particles. The authors have applied synchrotron-based scanning transmission soft $\mathrm{x}$-ray spectromicroscopy to image thin lipid layer patches with bound microspheres coated by a charged monolayer. The ability and current limits of scanning transmission x-ray spectromicroscopy to examine samples under physiologically relevant conditions in the presence of excess water have been tested. In particular, the authors have investigated a range of model lipids and have shown that these can be reproducibly identified from the near-edge $\mathrm{x}$-ray absorption fine structure spectra at the carbon $K$ absorption edge. Reference spectra were obtained based on a compact laser-driven plasma source, while the spectromicroscopy data were collected using synchrotron radiation at a lateral resolution of about $60 \mathrm{~nm}$. The authors show that thin lipid layer sensitivity can indeed be reached under physiological conditions and that membrane colloid interaction as well as eventual lateral segregation of lipid components may be probed in the future by this technique. (0) 2008 American Vacuum Society. [DOI: 10.1116/1.2976445]
\end{abstract}

\section{INTRODUCTION}

A quantitative understanding of the interactions between nanoparticles and biological interfaces, in particular, the cell membrane, is a prerequisite for the design of drug delivery systems based on synthetic nanoscale carrier systems, as well as for imaging agents such as fluorescent quantum dot markers. Functionally relevant issues are related to the binding affinity, the range of interaction, possible toxic effects due to membrane disruption or lysis, ${ }^{1-3}$ as well as the lateral reorganization of a multicomponent membrane in response to nanoparticle binding. ${ }^{4}$ The dominant interaction forces between nanoparticles and membranes are electrostatic in nature since charged lipids are common and versatile constituents of biological membranes. Charges in such lipids differ in magnitude and location within the interface and can locally demix in response to charged nanoparticles. ${ }^{5}$ If binding of nanoparticles to a multicomponent membrane results in a redistribution of charged and uncharged lipids, this altered membrane state will in return affect the bilayer-nanoparticle interaction, so that strong nonlinear effects can be expected. From a fundamental point of view, the interaction between two classical model systems, the lipid bilayer on one side

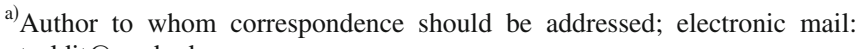
tsaldit@gwdg.de and colloidal nanoparticles on the other, can serve as a wellcontrolled testing ground for concepts in colloid science and soft condensed matter physics.

These important topics can be addressed using a solid supported phospholipid membrane as a biomimetic model for nanoparticle-phospholipid interactions since geometry, composition, and environmental parameters can be better controlled in a biomimetic system than in a cell. ${ }^{6}$ Moreover, planar bilayer systems are amenable to quantitative high resolution analytical techniques, which cannot or not yet be applied to real biological membranes. The biophysical techniques carried out on planar bilayers range from infrared spectroscopy, plasmon resonance, x-ray and neutron reflectivities, to fluorescence microscopy. Many of these involved charged species. The absorption of highly charged biomolecules such as DNA with the cationic and mixed bilayers on solid surfaces has been studied by fluorescence microscopy. ${ }^{7}$ The structure of two-component lipid mixtures, with varied surface charges, both in the fluid and gel phases has been probed by using x-ray reflectivity. ${ }^{8}$

A particular technique which may be useful to such systems but which has to date not been fully exploited, not even for studies of pure lipid membranes, is synchrotron-based soft x-ray microscopy and spectromicroscopy. This technique can provide the required combination of chemical sensitivity and high spatial resolution, in particular, to probe the 
lateral organization of a multicomponent membrane. In nearedge x-ray absorption fine structure (NEXAFS) spectromicroscopy, inner-shell electronic excitations ${ }^{9}$ are used as a chemically sensitive image-contrast mechanism. Hitchcock et al. $^{10}$ showed that while scanning transmission $\mathrm{x}$-ray microscopy is essentially a "bulk" technique, protein monolayer detection is possible, although close to the current limit of sensitivity.

In this work we have used scanning transmission x-ray microscopy to image positively charged polystyrene latex microspheres which bind onto a supported two-component lipid membrane, composed of charged and neutral lipid species. We used protocols for the deposition of solid supported lipid bilayers by vesicle fusion, ${ }^{11}$ which have been adapted for deposition of charged bilayers. ${ }^{12}$ Carbon $K$-edge NEXAFS spectroscopy is particularly favorable for studies of phospholipids since they have a strong $\pi^{*}(\mathrm{C}=\mathrm{O})$ resonance at $\sim 288.5 \mathrm{eV}^{13}$ We have tested the ability of scanning transmission $\mathrm{x}$-ray spectromicroscopy to examine samples under physiologically relevant conditions in the presence of excess water. In the energy range of the so-called water window between oxygen and the carbon $K$ absorption edges (283 and $543 \mathrm{eV}$ ), specimens in water show a natural absorption contrast, which permits imaging of unstained specimens in a hydrated state. The samples are then imaged both below and above the carbon $K$ absorption edge. The amount of the carbon can be determined quantitatively from the difference in the x-ray absorptions. The thickness of the lipid layer and the microspheres can then be determined. Spectromicroscopy data can be extended to a sequence of images over a series of spectroscopically interesting energies, providing spatially and spectrally resolved data of the sample simultaneously. ${ }^{14}$ Such image stacks have been collected on dried colloids as well as wet lipid membrane/polystyrene microspheres.

A further, more technical motivation for the present study was to demonstrate the feasibility of lipid thin films carbon $K$-edge NEXAFS experiments with x-ray radiation generated from compact sources based on a laser-produced plasma. NEXAFS experiments have almost exclusively required synchrotron radiation before. Peth et al. ${ }^{15}$ recently presented NEXAFS results that were obtained by using a laboratory scale laser-driven plasma source. In this work, we show the NEXAFS spectra of selected lipids, which differ in the headgroup structure or/and in containing the $\mathrm{C}=\mathrm{C}$ double bond in hydrocarbon tails. $\pi^{*}(\mathrm{C}=\mathrm{C}), \pi^{*}(\mathrm{C}-\mathrm{C}), \sigma^{*}(\mathrm{C}=\mathrm{C}), \sigma^{*}(\mathrm{C}-\mathrm{C})$, and Rydberg resonances and $\mathrm{C} 1 s \rightarrow \sigma^{*}(\mathrm{C}-\mathrm{N})$ transitions were resolved.

The article is organized as follows: After this Introduction, the experimental methods and materials are described in Sec. II along with some characterization of the planar lipid bilayers by more conventional techniques. Section III presents the results of the measurements, followed by a short summary and conclusion section.

\section{EXPERIMENTAL METHOD}

\section{A. Sample preparation}

1,2-dipalmitoyl-sn-glycero-3-phosphatidilcholine

(DPPC), 1,2-dipalmitoyl-sn-glycero-3-phosphatidilserine (DPPS), 1,2-dioleoyl-sn-glycero-3-phosphatidilcholine (DOPC), 1,2-dioleoyl-sn-glycero-3-phosphatidilserine (DOPS), and 1,2-dimyristoyl-3-phosphatidylcholin (DMPC) were obtained from Avanti Polar Lipids (Alabaster, AL). Hepes (H-4034), $\mathrm{NaCl}$ (S-9888), and chloroform (25693) were purchased from Sigma-Aldrich (Germany), $\mathrm{CaCl}_{2}$ from Merck KGaA (Germany), and the positively charged polystyrene (PS) latex particles (amidine charge groups) from Interfacial Dynamics Corp. (Portland, OR). Latex colloids are reported by the manufacturer to have a mean radius of $0.195 \mu \mathrm{m}$ and a density of $1.055 \mathrm{~g} / \mathrm{cm}^{3}$. Lipid bilayers were prepared on cleaned transparent $\mathrm{Si}_{3} \mathrm{~N}_{4}$ membranes [the frames $\left(10 \times 10 \mathrm{~mm}^{2}\right)$ which consist of a thin window with membrane of $100 \mathrm{~nm}$ thickness and $5 \mathrm{~mm}^{2}$ membrane size], supplied by Silson Ltd, and $15 \times 10 \mathrm{~mm}^{2}$ (100)-silicon wafers (Silchem, Germany). Lipid bilayers were prepared by vesicle fusion method. ${ }^{12}$ The $\mathrm{Si}_{3} \mathrm{~N}_{4}$ windows and the silicon wafers were cleaned by two 20 min cycles in methanol, followed by two 20 min cycles in ultrapure water $(18 \mathrm{M} \Omega \mathrm{cm}$, Millipore, Bedford, MA), and drying under nitrogen flow. Finally, the surface of the windows and the wafers were rendered hydrophilic and cleaned of organic contaminants by etching in a plasma cleaner (Harrick Plasma, NY) for 3 min.

A buffer solution (A), made of $100 \mathrm{mM} \mathrm{NaCl}$ and $10 \mathrm{mM}$ Hepes, $p \mathrm{H} 7.4$, was prepared in ultrapure water and a buffer (B) was prepared by adding $2 \mathrm{mM} \mathrm{CaCl}_{2}$ to the buffer (A).

DPPC and DPPS lipids were mixed in the desired amount (molar ratio 4:1) and dissolved in chloroform. The solvent was evaporated, followed by drying in a vacuum oven for 24 $\mathrm{h}$ in order to remove all traces of solvent. The lipid mixture was resuspended in a buffer solution at total concentrations of $0.75 \mathrm{mg} / \mathrm{ml}$. Suspension was vortexed. Unilamellar vesicles were obtained by sonicating lipid solutions with a tip sonicator (Sonoplus, Germany) for $15 \mathrm{~min}$, followed by centrifugation in an Eppendorf centrifuge $(10 \mathrm{~min}$ at $14.000 \mathrm{~g}$ ) to remove titanium particles.

The drop of lipid vesicles, prepared in the buffer solution (A), was placed over cleaned $\mathrm{Si}_{3} \mathrm{~N}_{4}$ window and incubated at $55^{\circ} \mathrm{C}$ for $30 \mathrm{~min}$. Excess vesicles were rinsed away, while the lipid membrane remained hydrated all the time during preparation and measurement. To this mixture, we added positively charged PS latex microspheres at volume fractions of about $0.04 \%$. After a $30 \mathrm{~min}$ incubation, the unbound microspheres were gently rinsed off with the buffer solution. The prepared sample was capped by a second $\mathrm{Si}_{3} \mathrm{~N}_{4}$ window to form a wet cell (Fig. 1). The wet cell was sealed and glued onto a metal shim. The shim was then fixed between the two specimen chamber rings. The specimen chamber was designed to be used with the scanning transmission X-ray microscope at BESSY II. ${ }^{16}$ 


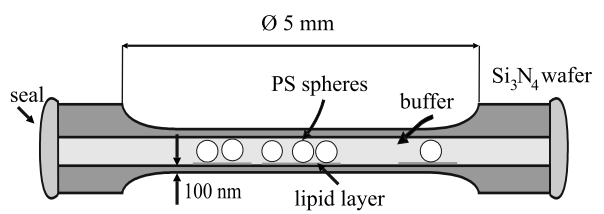

FIG. 1. Schematic of the measurement cell.

A sample of air-dried PS microspheres with radii of 195 $\mathrm{nm}$ was prepared. The droplet of colloid solution was deposited on the $\mathrm{Si}_{3} \mathrm{~N}_{4}$ membrane and allowed to dry out on the surface.

Lipid multilayer samples were prepared on $\mathrm{Si}_{3} \mathrm{~N}_{4}$ windows. Lipids were dissolved in chloroform at total concentrations of $5 \mathrm{mg} / \mathrm{ml}$. A drop of $1,0.5$, or $0.01 \mu \mathrm{l}$ was then carefully spread onto well-leveled and cleaned $\mathrm{Si}_{3} \mathrm{~N}_{4}$ windows. The spread solution was allowed to dry. Finally, the films were exposed to high vacuum over $24 \mathrm{~h}$ to remove all traces of solvent.

A sample for x-ray reflectivity measurement was prepared on a cleaned silicon wafer placed into a Teflon chamber with Kapton windows. The chamber was filled with DPPC:DPPS (4:1) lipid vesicles, prepared in the buffer solution (B), and incubated for $30 \mathrm{~min}$. Excess vesicles were rinsed away.

\section{B. Intrumentation and data acquisition: The scanning transmission $\mathrm{X}$-ray microscope at BESSY II}

The experiment was carried out both with the scanning transmission $\mathrm{x}$-ray microscope (STXM) at the undulator beamline U41-STXM (BESSY II, Berlin) ${ }^{17}$ and at a laserdriven soft X-ray plasma source in the water window based on a pulsed krypton gas target. ${ }^{15}$ The design of the STXM (see Fig. 2) is described in detail in Ref. 18. The undulator U41 provides high brilliance $\mathrm{x}$ rays which pass through the monochromator to select a narrow photon energy range. The resulting maximum energy resolution with the STXM is about $0.18 \mathrm{eV}$ at $300 \mathrm{eV}$. A Fresnel zone plate ${ }^{19}$ was used as a diffractive lens to form a high resolution focused spot of soft $\mathrm{x}$ rays. The Fresnel zone plate $(\mathrm{ZP})$ used here has a diameter of $200 \mu \mathrm{m}$, an outermost width of $50 \mathrm{~nm}$, number of zones of 1000 , and a central stop of $65 \mu \mathrm{m}$, with a theoretical Rayleigh resolution of $61 \mathrm{~nm}$ in the first diffraction order. An order-sorting aperture with a radius of $40 \mu \mathrm{m}$ is placed between the sample stage and zone plate stage. It selects the light of the first diffraction order to illuminate the sample and blocks the light from the zeroth and from higher orders. The sample is scanned in the $x, y$ plane by moving the ZP with a high resolution piezoscanner. The transmitted intensity through the sample is recorded on a single $p n$

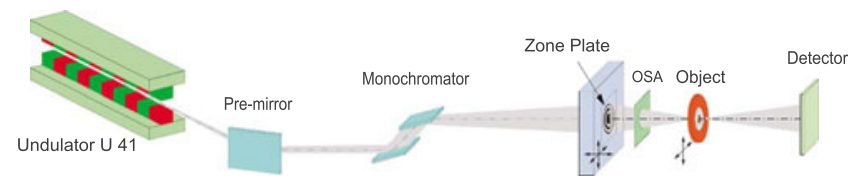

FIG. 2. Schematic overview of the BESSY II STXM (see text) (Ref. 18).

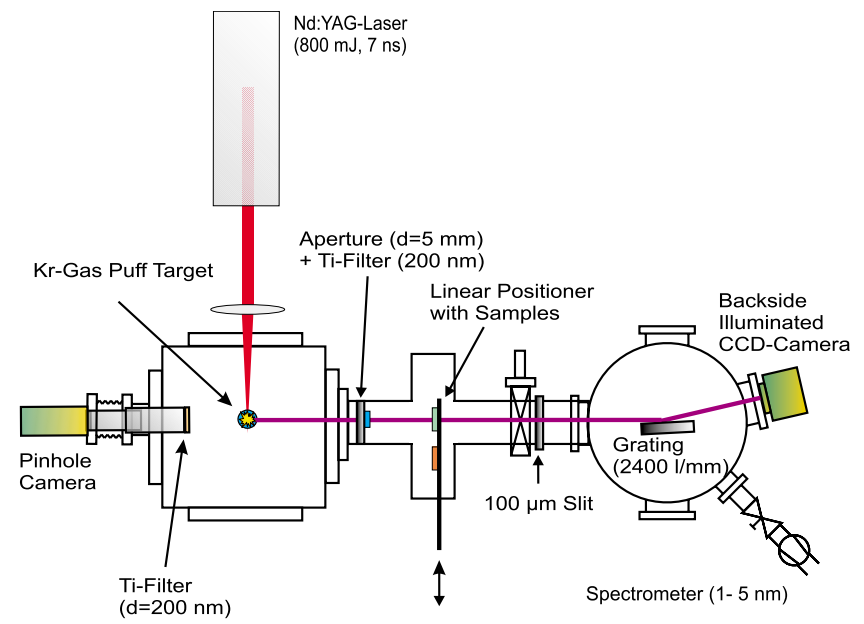

FIG. 3. Experimental setup of the laser plasma XUV source used for NEXAFS experiments.

charge coupled device $(C C D)$ unit $^{20}$ of $200 \times 64$ pixels with $150 \times 150 \mu \mathrm{m}^{2}$ pixel size. During experiment, the detector is kept at liquid-nitrogen temperature $(77 \mathrm{~K})$ and vacuum conditions of $3 \times 10^{-8}$ mbar to reduce the dark current.

The simplest method for x-ray absorption spectroscopy (XAS) is a transmission XAS experiment. The transmitted X-ray intensity $(I)$ through a material depends on the thickness, density, atomic number of the material, and energy of the individual photons. According to the Beer-Lambert law,

$$
I(E)=I_{0}(E) \exp (-\mu(E) d),
$$

where $I_{0}$ is the incident intensity and $d$ is the material thickness. $\mu$ is the linear absorption coefficient in units of 1/length, which can be expressed for a single element as

$$
\mu=2\left(N_{A} / M\right) r_{e} \rho \lambda f_{2}(\lambda),
$$

where $N_{A}$ is Avogadro's number, $M$ is the molar mass, $r_{e}$ $=2.818 \times 10^{-15} \mathrm{~m}$ is the classical radius of the electron, $\rho$ is the element's density, $\lambda$ is the $\mathrm{x}$-ray wavelength, and $f_{2}(\lambda)$ is the imaginary part of the complex scattering factor, tabulated for all relevant elements in the soft $\mathrm{x}$-ray energy range. ${ }^{21}$ Compounds and molecules can be modeled, away from the resonances, by calculating $f_{2} / M$ as a weighted sum over all elements. The intensity absorption is also commonly expressed by the mass attenuation coefficient $\mu_{m}=\mu / \rho$. In XAS, the dependence of the absorption coefficient $\mu$ on the wavelength of the incoming $\mathrm{x}$-ray beam, the so-called optical density $\left[\mathrm{OD}=\mu d=-\ln \left(I / I_{0}\right)\right]$, is measured.

Spectral properties of the sample constituents also form physical bases for a contrast mechanism in a direct $\mathrm{x}$-ray image formation. Combining this $\mathrm{x}$-ray spectral fingerprint with direct high resolution $\mathrm{x}$-ray imaging is termed NEXAFS spectromicroscopy.

\section{Laboratory scale XUV source}

The experimental setup for soft $\mathrm{x}$-ray radiation emitted from laser plasmas (see Fig. 3) consists of a neodymium doped yttrium aluminum garnet laser beam (Innolas, 1064 
$\mathrm{nm}, 1 \mathrm{~Hz}, 800 \mathrm{~mJ}, 7 \mathrm{~ns}$ ) focused into a pulsed krypton gas jet centered in a vacuum chamber. ${ }^{22}$ The laser focus has a diameter of about $60 \mu \mathrm{m}$, yielding power densities of up to 4 $\times 10^{12} \mathrm{~W} / \mathrm{cm}^{2}$. The size of the krypton plasma is about $250 \mu \mathrm{m}$ (full width at half maximum) in the horizontal and $150 \mu \mathrm{m}$ in the vertical direction. An XUV spectrometer $(1-5 \mathrm{~nm})$ was mounted $90^{\circ}$ to the laser beam. The spectrometer consists of a $100 \mu \mathrm{m}$ entrance slit, an aberration corrected flat-field grating (Hitachi, 2400 lines/mm), and a back side illuminated CCD camera (Roper Scientific, pixel size of $13 \mu \mathrm{m})$. The resolution was $\lambda / \delta \lambda \approx 200$ at $2.87 \mathrm{~nm}$. To block visible radiation from the plasma and scattered laser light, a titanium foil (200 $\mu \mathrm{m}$ thickness) was positioned between the plasma source and the sample. Due to the small mean free path of the soft $\mathrm{x}$-ray radiation at atmospheric pressure the target vacuum chamber is evacuated to approximately $10^{-4}$ mbar. For adjustment in the XUV beam the samples were mounted on a rotary/linear motion stage. The distance between the plasma source and the sample is about $220 \mathrm{~mm}$ and the distance between the sample and the entrance slit of the spectrometer is about $425 \mathrm{~mm}$.

To obtain NEXAFS spectra with a high signal-to-noise ratio the data acquisition was accumulated over 1000 pulses (total acquisition time of about $16 \mathrm{~min}$ ). Since it was not possible to record the transmitted intensity through the sample and the reference spectra simultaneously, they were taken consecutively. In previous experiments the stability of the laser plasma source was tested for corresponding acquisition times and it was found that the deviation between the spectra was less than 5\%. Since the laser plasma source emits radiation into $4 \pi$ (in steradian), the sample was fully illuminated. Nevertheless, only radiation transmitted through an area of $100 \mu \mathrm{m} \times 2.3 \mathrm{~mm}$ of the sample is collected by the spectrometer since the entrance slit in combination with the detector height is the limiting aperture.

\section{X-ray reflectivity measurement}

X-ray reflectivity experiment was performed at the ID01 undulator beamline (ESRF, Grenoble) using a $13.46 \mathrm{keV}$ photon energy. The intensity of scattered $\mathrm{x}$ rays was measured as a function of an incident angle $\alpha_{i}$ under specular condition (exit angle $\alpha_{f}=\alpha_{i}$ and out-of-plane angle $2 \theta=0$ ). The momentum transfer of the elastic scattering $\vec{q}$ was always along $q_{z}$, with the $z$ axis parallel to the sample normal. Additionally, scans of the x-ray intensity offset from the specular condition (by moving the detector to $\alpha_{f} \neq \alpha_{i}$ ) were made in order to correct the data for diffuse scattering. A more detailed description of the reflectivity experiment can be found in Ref. 8 .

\section{E. Atomic force microscopy and optical imaging profiler}

Atomic force microscopy (AFM) was performed on a NanoWizard AFM (JPK Instruments, Berlin, Germany) operated in tapping mode in water. Ultrasharp (NT-MDT, Moscow, Russia) silicon tips were used.
The surface topography of lipid multilayers was characterized by the PL $\mu 2300$ optical imaging profiler (Sensofar Tech S.L., Spain). Images were taken in the phase shifting interferometer modus.

\section{F. Characterization of DPPC:DPPS (4:1) lipid membranes}

The process of supported lipid bilayer formation can be generally divided into a number of steps: adhesion, vesicle flattening, rupture, and formation of a planar single-bilayer structure. ${ }^{23,24}$ In the first step the bound vesicle is believed to collapse onto itself, resulting in a double-bilayer structure. This process continues until the entire vesicle transforms to two bilayers stacked on top of each other. ${ }^{23}$ The transition to a single bilayer takes place in a way that may be interpreted as a sliding or rolling motion. ${ }^{12,25}$ The deposition of vesicles composed of charged and zwitterionic lipid species was found to be enhanced in the presence of calcium ions. ${ }^{12}$ This is most likely due to the ability of $\mathrm{Ca}^{2+}$ ions to bridge negatively charged entities such as the carboxylate groups and the phosphate groups in charged and zwitterionic lipid species or negative charges on the silica surface. The most important effect of $\mathrm{Ca}^{2+}$ was suggested to be due to the well-known fact that this ion is a strong fusogenic agent, promoting the fusion of cells and liposomes. ${ }^{26}$

We prepared a negatively charged DPPC/DPPS (4:1) supported lipid membrane on the silicon support in the buffer containing $\mathrm{Ca}^{2+}$ ions. The lipid membrane was characterized by specular x-ray reflectivity. The fit to the data was determined by obtaining model intensity curve using the semikinematical approach described in Ref. 8. Structural parameters, such as membrane thickness, area per lipid, water thickness, and number of water molecules per lipid, were calculated directly from an electron density profile. We obtained the electron density of lipid heads of $\rho_{h}=0.44 e^{-} / \AA^{3}$ and the electron density of lipid tails of $\rho_{t}=0.2 e^{-} / \AA^{3}$. The thickness of the bilayer was found to be $d_{h h} \sim 4.5 \mathrm{~nm}$. The obtained average area per lipid was $A=43.3 \pm 2.6 \AA^{2}$. The values are in good agreement with the results obtained for the gel phase DPPC bilayer by using $\mathrm{x}$-ray scattering methods. ${ }^{27}$ The water layer between the silicon and DPPC/ DPPS (4:1) was found to be $\sim 5.7 \AA$. The measured data, the fit, and the resulting electron density profile are shown in Fig. 4(a).

To ensure that $\mathrm{Ca}^{2+}$ ions will not partially screen electrostatic interactions between anionically charged lipids and positively charged PS microspheres, we have prepared a DPPC/DPPS (4:1) supported lipid layer in the buffer without $\mathrm{Ca}^{2+}$ ions. The vesicle fusion then results in an incomplete coverage shown in Fig. 4(b). The lipid layer thickness was measured to be $\sim 10 \mathrm{~nm}$, which might indicate the intermediate step in a single-bilayer formation process, two bilayers stacked on top of each other. Incomplete coverage was found to be an advantage for scanning transmission $\mathrm{x}$-ray spectromicroscopy experiments because of lateral contrast variation.

Colloids are able to induce bending deformations in charged membranes, giving rise to various phenomena such 
a)
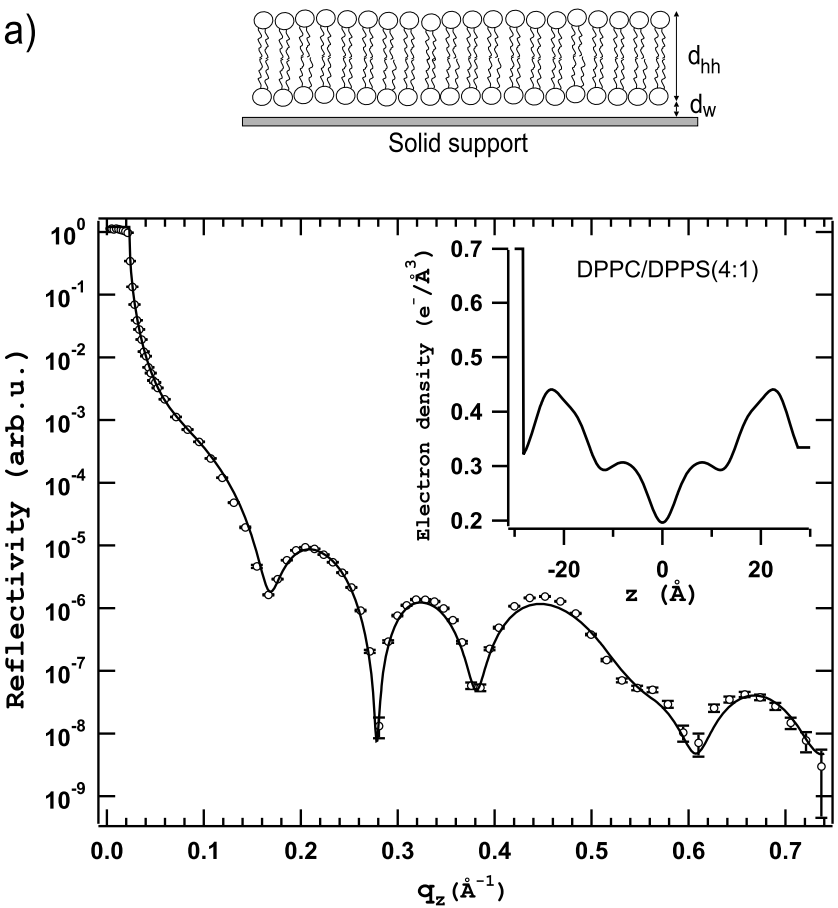

b)

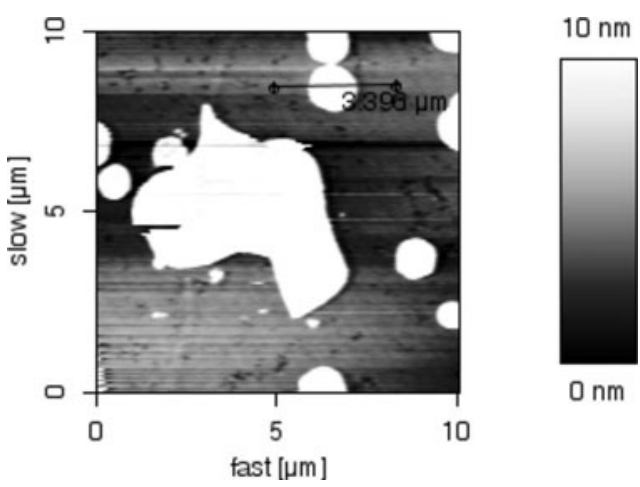

FIG. 4. (a) The fit (solid lines) to the reflectivity curve of DPPC/DPPS(4:1) in the gel phase at $23.8{ }^{\circ} \mathrm{C}$ obtained under complete wetting conditions, recorded at the ID01 beamline. The inset shows the electron density profile corresponding to the simulation. (b) The AFM image of the incomplete DPPC/DPPS(4:1) layer, which is obtained in the buffer without $\mathrm{Ca}^{2+}$ ions [as opposed to (a)], used to study the interaction with PS microspheres.

as partial or full wrapping of the membrane around colloids. ${ }^{4,28}$ Electrostatic adsorption of colloids on a twocomponent membrane with ideal mixing properties can lead to compositional inhomogeneities at the colloid adsorption sites. ${ }^{4}$ Schematic illustration of possible colloids induced lipid membrane deformation and microdomain formation is shown in Fig. 5.

\section{RESULTS}

\section{A. Elemental contrast imaging}

Using the STXM for absorption difference imaging was performed by taking two images of the same area at either side of the carbon $K$ absorption edge, at $E_{1}$ and $E_{2}$, respectively. The carbon map is found by taking the log of the ratio a)
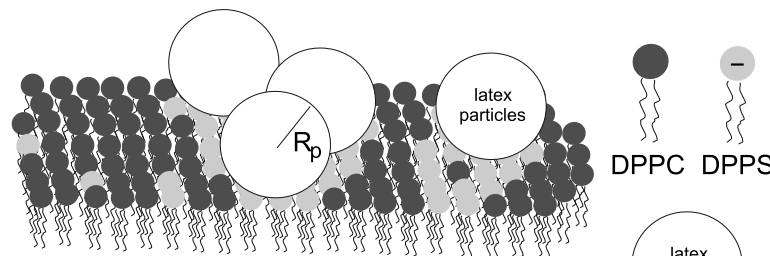

DPPC DPPS

b)
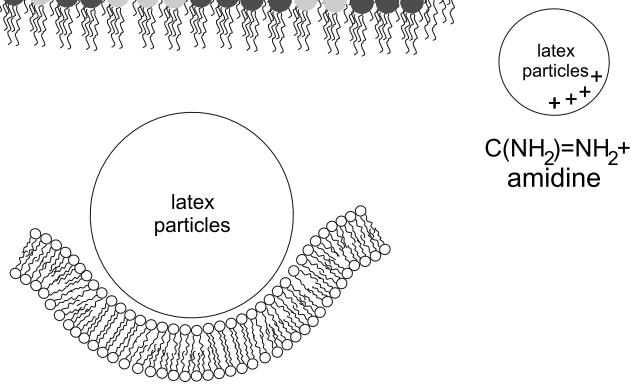

c)

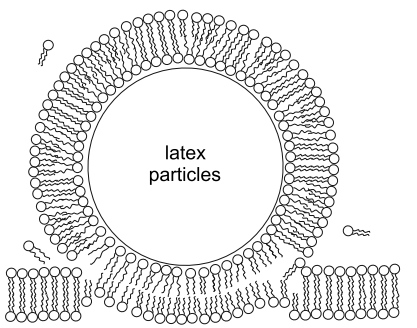

$\mathrm{C}\left(\mathrm{NH}_{2}\right)=\mathrm{NH}_{2}+$

amidine

FIG. 5. Schematic illustration of charged, two-component lipid membrane consisting of neutral and negatively charged lipids. Electrostatic binding to oppositely charged microspheres can induce the formation of (a) microsphere-coupled microdomains and/or bending deformations in the charged membrane resulting in (b) partial or (c) full wrapping of the membrane around microspheres.

of the corresponding pixel counts. This results in an image that when displayed indicates increasing carbon concentration by an increasing brightness level. ${ }^{29}$

Figure 6 shows $\mathrm{x}$-ray micrographs of the wet specimen composed of a supported DPPC/DPPS (4:1) lipid layer in the gel phase binding amidine functionalized polystyrene microspheres with radii of $195 \mathrm{~nm}$. An image of the region of interest below $\left(E_{1}=284 \mathrm{eV}\right.$, top $)$ and above $\left(E_{1}=314 \mathrm{eV}\right.$, center) the carbon $K$ edge were taken. The darker the color, the less intense is the measured signal. The quotient image (Fig. 6, bottom) shows the distribution of carbon. The image of a different region of the sample and magnified images of the selected area taken at 284 and $314 \mathrm{eV}$ with the corresponding carbon map image are shown in Fig. 7. Periodic (moiré) noise in all images is presumably due to aliasing (beating) of a systematic noise signal with the scan frequency of the STXM.

Away from an absorption edge, the linear absorption coefficient has an $E^{-3}$ dependence. Therefore, we can estimate the average thickness of a certain compound in selected pixels as

$$
d_{\text {comp }}=\frac{\ln \left(I_{01} / I_{1}\right)-\left(\lambda_{1} / \lambda_{2}\right)^{3} \ln \left(I_{02} / I_{2}\right)}{\mu_{1}-\mu_{2}\left(\lambda_{1} / \lambda_{2}\right)^{3}},
$$

where $I_{01}$ and $I_{02}$ are the intensities of the incident x-ray beams, $I_{1}$ and $I_{2}$ are the intensities of the transmitted x rays, 

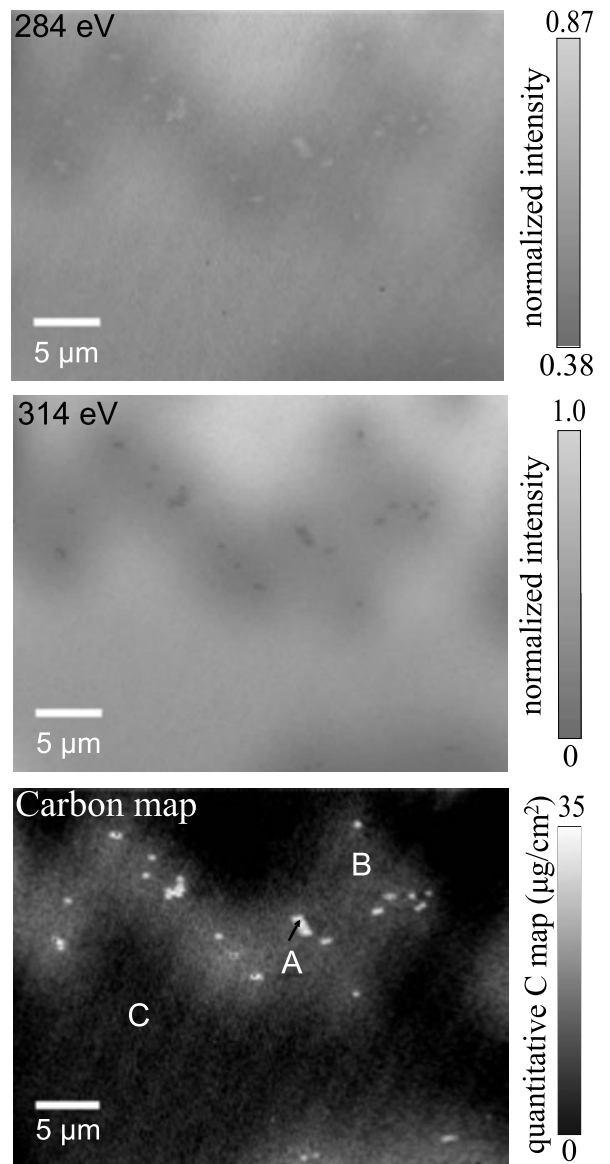

FIG. 6. Elemental imaging at the carbon $K$ absorption edge from the lipid layer/PS microsphere sample in the wet state on a $\mathrm{Si}_{3} \mathrm{~N}_{4}$ membrane. Shown are absorption contrast images (a) below the absorption edge at $284 \mathrm{eV}\left(I_{1}\right.$, top) and above the absorption edge at $314 \mathrm{eV}\left(I_{2}\right.$, center). The carbon map (bottom) is calculated from the top and the center image. The grayscale bar indicates the amount of carbon. The arrow ("A") in the carbon map image indicates an amidine coated PS latex microspheres. "B" indicates a lipid layer. The images have a $37 \times 29 \mu \mathrm{m}^{2}$ size, $200 \mathrm{~nm}$ pixel size, and $6 \mathrm{~ms}$ pixel dwell time. The image acquired at $284 \mathrm{eV}$ has a higher relative noise level because of the lower photon rate.

and $\mu_{1}$ and $\mu_{2}$ are the linear absorption coefficients at $E_{1}$ and $E_{2}$, respectively. ${ }^{30}$

Pixels corresponding to polystyrene latex microspheres $\left(\mathrm{C}_{8} \mathrm{H}_{8}\right)$ with a density of $1.055 \mathrm{~g} \mathrm{~cm}^{-3}$ were selected [region A in Fig. 6(c)]. Linear absorption coefficients of the polystyrene were calculated from Eq. (2) to be $\mu_{P 1}=0.18 \mu \mathrm{m}^{-1}$ and $\mu_{P 2}=4.0 \mu \mathrm{m}^{-1}$ at $E_{1}$ and $E_{2}$, respectively. The thickness of the selected region A was estimated to be $0.33 \pm 0.09 \mu \mathrm{m}$. The error in the measured thickness is $\sim 30 \%{ }^{29}$

The selected region B in Fig. 6(c) should correspond to the DPPC/DPPS (4:1) lipid layer in the gel phase with a density of $1.25 \pm 0.08 \mathrm{~g} \mathrm{~cm}^{-3}$. The density was estimated from the values of the area per lipid in the mixture, $A$ $=43.3 \pm 0.25 \AA^{2}$, thickness of the membrane $d=45 \pm 0.5 \AA$, and molecular weights (MWs) $\mathrm{MW}(\mathrm{DPPC})=734.05$ and MW(DPPS $)=757.96$. Linear absorption coefficients of the lipid mixture according to Eq. (2) are $\mu_{L 1}=0.49 \mu \mathrm{m}^{-1}$ and $\mu_{L 2}=3.7 \mu \mathrm{m}^{-1}$ at $E_{1}$ and $E_{2}$, respectively. The thickness of region $B$ was calculated to be $14 \pm 4.2 \mathrm{~nm}$.

The water thickness in the specimen was estimated from region $\mathrm{C}$ in Fig. 6(c). The flux $I_{0}$ behind two $\mathrm{Si}_{3} \mathrm{~N}_{4}$ foils without the specimen at the pre-edge energy of $284 \mathrm{eV}$ was $5.4 \times 10^{5}$ photons $/ 6 \mathrm{~ms}$. The flux $I$ behind the wet specimen sandwiched between two $\mathrm{Si}_{3} \mathrm{~N}_{4}$ foils in region $\mathrm{C}$ at 284 $\mathrm{eV}$ was $4.3 \times 10^{5}$ photons $/ 6 \mathrm{~ms}$. The water thickness was calculated from $t_{\text {water }}=-\ln \left(I / I_{0}\right) / \mu_{\text {water }}$ to be $430 \mathrm{~nm}$.

The grayscale bar in Fig. 6(c) indicates the carbon mass density of $m_{C}=\rho_{C} d_{C}$, where the carbon density $\rho_{C}$ is assumed to be $1.05 \mathrm{~g} \mathrm{~cm}^{-3}$ and $d_{C}$ is calculated using Eq. (3).

\section{B. Spectromicroscopy of dried amidine functionalized polystyrene particles}

A method to obtain spectromicroscopic data using STXM is to acquire an image sequence over a series of spectroscopically interesting energies. ${ }^{31}$ The images in this sequence can then be correlated with each other and shifted to provide the proper alignment. A spectrum is obtained from each selected object point by plotting the OD of all energies whose images have been taken. In this way, an image is obtained at each energy, and also a spectrum over the entire energy range can be extracted from each pixel or from larger regions. A beam shutter was used to block the beam during energy changes. Refocusing after a change in energy was necessary.

The measurement was performed on air-dried amidine functionalized PS microspheres with radii of $195 \mathrm{~nm}$ deposited on the $\mathrm{Si}_{3} \mathrm{~N}_{4}$ membrane. A sequence of $72 \mathrm{x}$-ray micrographs $\left(10 \times 10 \mu \mathrm{m}^{2}\right)$ at a spatial resolution of $100 \mathrm{~nm}$ per pixel ( $6 \mathrm{~ms}$ dwell time per pixel) with energy steps of 0.25 $\mathrm{eV}$ over the energy range of $278-296 \mathrm{eV}$ was taken. The images have to be aligned with each other using a cross-
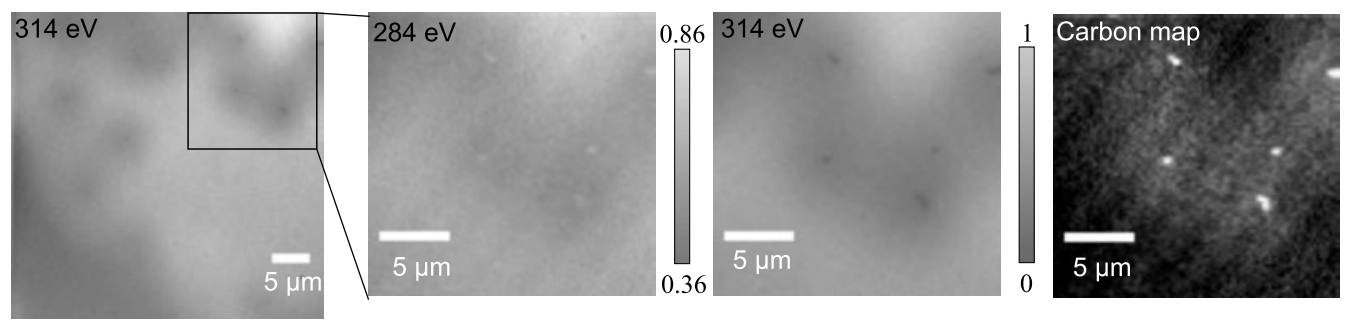

Fig. 7. (Left) Image of the lipid layer/PS microsphere sample in the wet state. Magnified images of the selected area were taken at 284 and $314 \mathrm{eV}$, and the carbon map image was created (right). Images have $18 \times 18 \mu \mathrm{m}^{2}$ size, $200 \mathrm{~nm}$ pixel size, and $6 \mathrm{~ms}$ pixel dwell time. 
correlation method described by Jacobsen et al. ${ }^{31}$ and the PS spectrum from the region of interest was extracted by using a package of stack analyze routines. ${ }^{31}$

The presence of PS microspheres was revealed through a change in contrast, i.e., high transmission at $278 \mathrm{eV}$ and strong absorption at $285 \mathrm{eV}$ [Fig. 8(a)] corresponding to the $\pi^{*}(\mathrm{C}=\mathrm{C})$ resonance. The shadowing effect to the right of each PS microsphere is an artifact of the detector electronics. ${ }^{18}$

In the carbon map, the distribution of organic carbon [last image in Fig. 8(a)] was obtained by taking the ratio of images acquired at different energies (278-283 versus 290-296 $\mathrm{eV}$ ) and mapping out the areas that exhibited the greatest change in absorbance just below the carbon absorption edge $(290 \mathrm{eV})$.

To extract the spectrum of PS microspheres from the image sequence, we selected pixels corresponding to the particles, $I$ region. The necessary incident flux $I_{0}$ was derived from the region in the images without an object structure [see Fig. 8(b)]. The spectrum shown in Fig. 8(c) was calculated as $\mathrm{OD}=-\ln \left(I / I_{0}\right)$ and plotted as a function of energy. The SPECFIT package ${ }^{32}$ was used to apply the Gaussian fitting for a more accurate determination of peak positions and an arctangent stepfunction at $290.1 \mathrm{eV}$ for the ionization continuum onset. The instrument was calibrated using the Rydberg lines of the well-known spectrum of $\mathrm{CO}_{2}$.

The spectrum of PS microspheres has a single, very intense peak at $285.1 \mathrm{eV}$ due to the $\pi^{*}(\mathrm{C}=\mathrm{C})$ resonance. The feature near $287.3 \mathrm{eV}$ is typically assigned to a Rydberg transition and the $288.9 \mathrm{eV}$ feature to a $\mathrm{C} 1 s(\mathrm{C}-\mathrm{H})$ $\rightarrow 2 \pi^{*}(\mathrm{C}=\mathrm{C})$ transition. Peak positions correspond well to NEXAFS data obtained from poly $(\alpha$-methyl styrene) by Dhez et al. ${ }^{33}$ The shoulder at $285.9 \mathrm{eV}$ (2) might correspond to the $\mathrm{C} 1 s(\mathrm{C}-\mathrm{N}) \rightarrow 1 \pi^{*}(\mathrm{C}=\mathrm{C})$ transition in amidine.

\section{Spectromicroscopy of the lipid layer with polystyrene microspheres}

A wet sample of a supported DPPC/DPPS (4:1) lipid layer in the gel phase binding amidine functionalized PS microspheres with radii of $195 \mathrm{~nm}$, prepared between two $\mathrm{Si}_{3} \mathrm{~N}_{4}$ membranes, was observed by STXM. An image sequence of $7 \times 7 \mu \mathrm{m}^{2} \mathrm{x}$-ray micrographs at a spatial resolution of 70 $\mathrm{nm}$ per pixel (6 ms dwell time per pixel) with energy steps of $0.25 \mathrm{eV}$ over the energy range of $278-297 \mathrm{eV}$ was acquired.

Figure 9(a) shows selected images from the sequence. Lighter shades correspond to a higher transparency. The absorption contrast reversal is clearly revealed. For example, at the pre-edge energy of $281.2 \mathrm{eV}$, water absorbs stronger than PS microspheres; hence they appear lighter at this energy. Strong absorption of PS spheres at $285 \mathrm{eV}$ corresponds to the $\pi^{*}(\mathrm{C}=\mathrm{C})$ resonance in PS.

The carbon map [Fig. 9(a), last image] obtained from the image sequence clearly shows lipid layer regions (region B). Lighter shades correspond to higher carbon concentrations.

Figures 9(b) and 9(c) present the spectra extracted from the spatially selected regions A (microspheres) and B (the lipid layer). The spectrum from region A is similar to that of
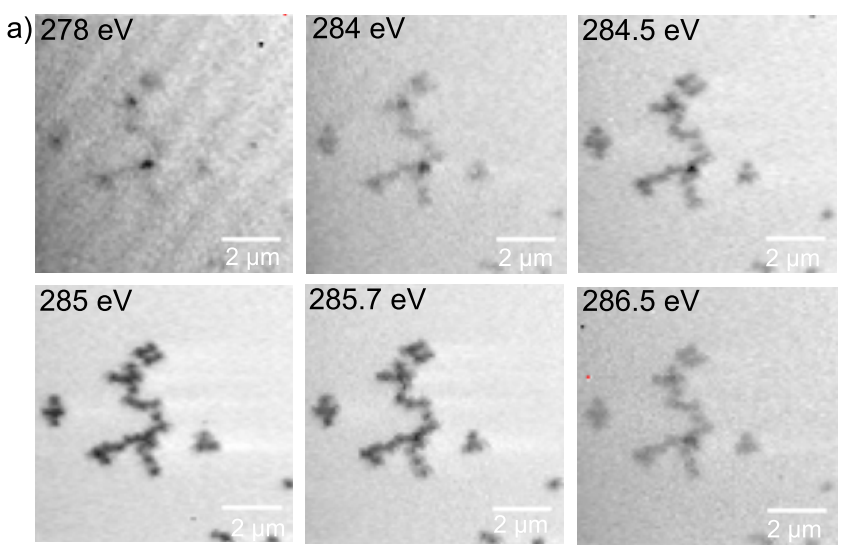

$286.5 \mathrm{eV}$
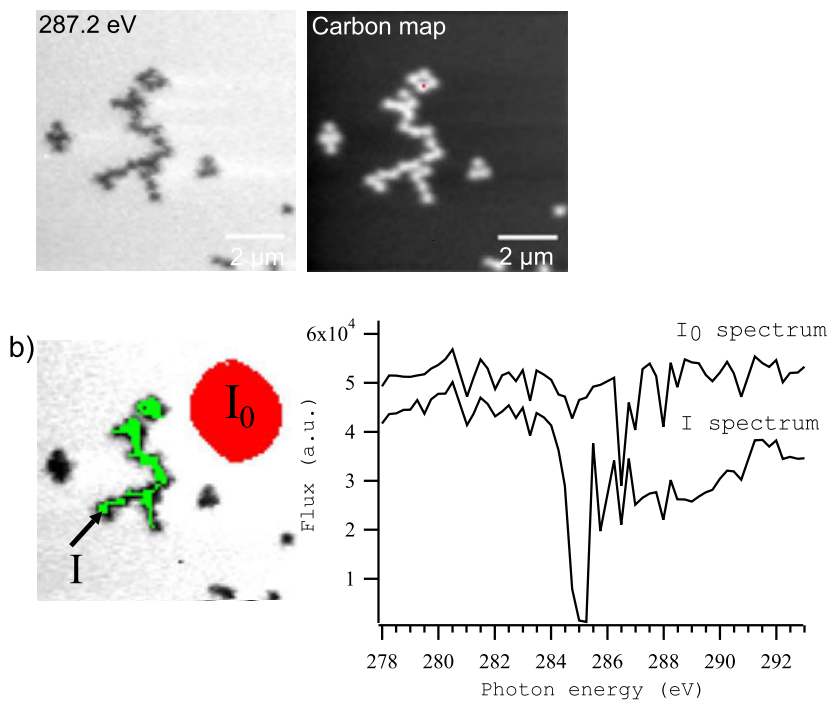

c)

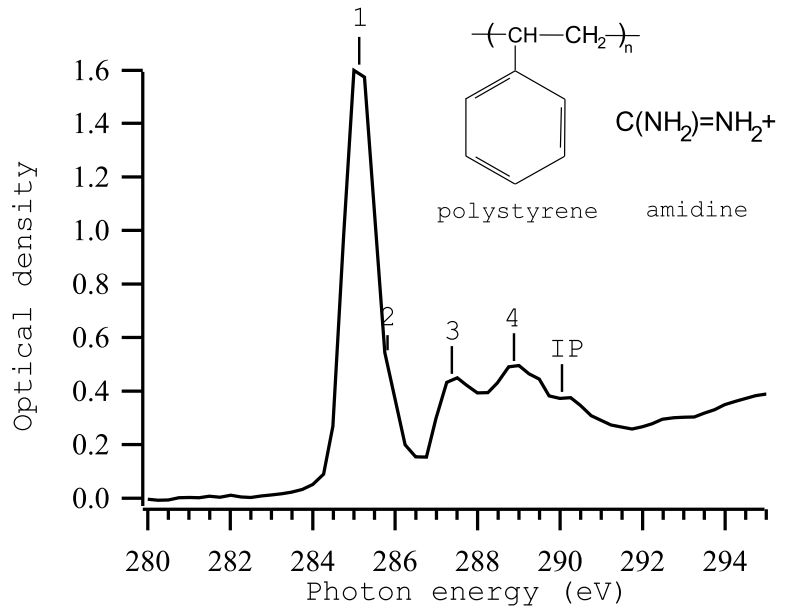

FIG. 8. (a) STXM images of the dried amidine PS particle sample at selected photon energies. (b) (Left) Selected regions for transmitted x-ray intensity $I$, incident X-ray intensity $I_{0}$, and corresponding $I$ and $I_{0}$ intensities plotted as a function of photon energy (right). (c) The derived spectrum $[\mathrm{OD}=$ $\left.-\ln \left(I / I_{0}\right)\right]$ at the carbon $K$ edge. The features of the spectrum are explained in the text.

dried PS except for an additional peak at $287.8 \mathrm{eV}$. This peak might be the $\pi^{*}(\mathrm{C}=\mathrm{O})$ resonance in the underlying lipid layer. The spectrum from region $\mathrm{B}$ shows a readily differentiated peak at $287.9 \mathrm{eV}$ [the $\mathrm{C} 1 s(\mathrm{C}=\mathrm{O}) \rightarrow \pi^{*}(\mathrm{C}=\mathrm{O})$ transition] typical of lipids. 


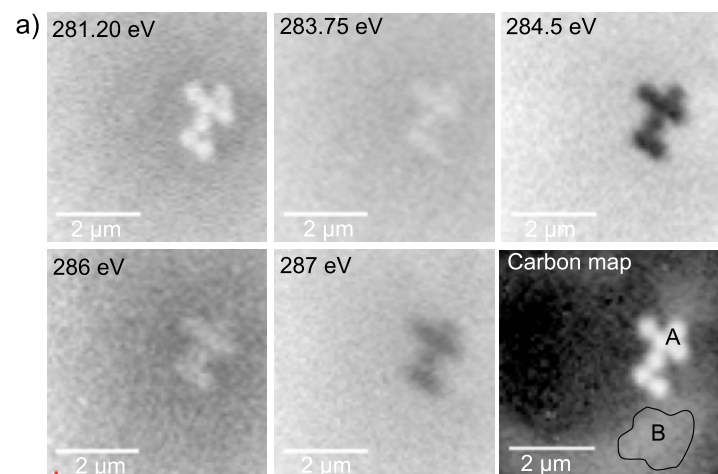

b)

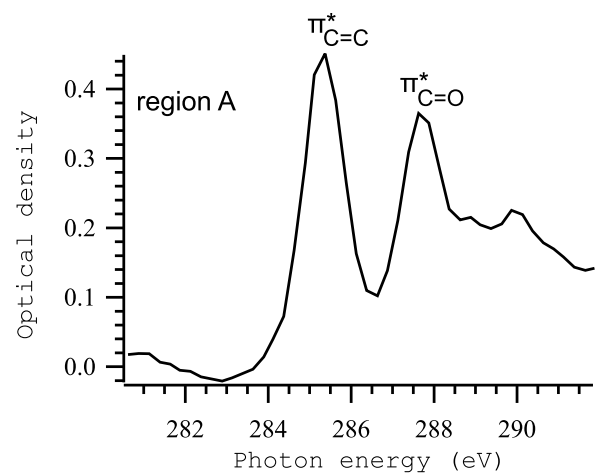

c)

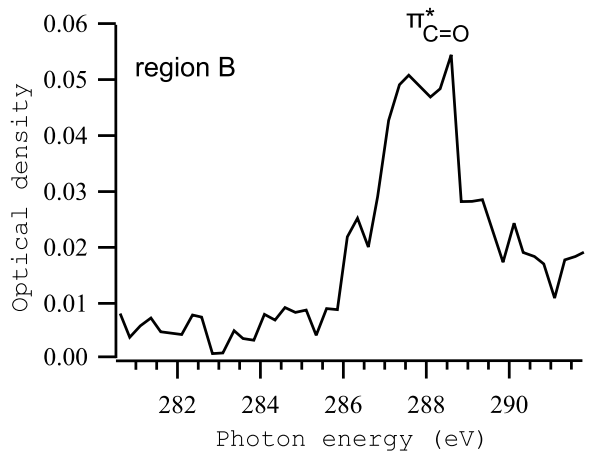

FIG. 9. (a) X-ray micrographs of the supported DPPC/DPPS (4:1) lipid layer in the gel phase binding amidine-PS microspheres at selected photon energies and the resulting carbon map image. [(b) and (c)] Spectra from spatially selected regions, corresponding to particles and the lipid layer.

The overlayer of the buffer is estimated from the pre-edge signal to be approximately $520 \mathrm{~nm}$ thick. Optical density associated with the lipid signal in region B is only $\sim 0.02$ in the continuum. This is equivalent to a lipid layer thickness of $5.5 \pm 2.7 \mathrm{~nm}$. The uncertainties in the quantification of the weak signal from the surface adsorbed organic layer is rather high - perhaps as much as $50 \%$. $^{34}$ Together these results constitute clear evidence that scanning transmission $\mathrm{x}$-ray microscopy can detect supported lipid layers in the aqueous environment.

In scanning transmission $\mathrm{x}$-ray microscopy the dosage applied to the specimen and the associated radiation damage, especially of biological specimen, plays an important role. Irradiation generates broken chemical bonds by excitations and more likely by ionizations of molecules. According to Ref. 35 we can estimate the radiation dose in gray as

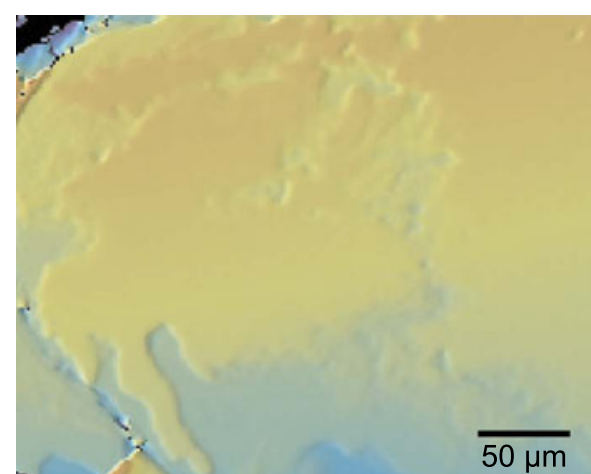

FIG. 10. DMPC supported multilayers (of $\sim 120 \mathrm{~nm}$ thickness) imaged in the dried state by optical imaging profiler.

$$
\text { dose }=1.602 \times 10^{-4} \frac{E N t \mu}{A \rho \eta},
$$

where $N$ is the number of absorbed photons per millisecond with the photon energy $E$ in $\mathrm{eV}$ and $t$ is the exposure time in milliseconds. A represents the size of the radiated area in $\mu \mathrm{m}^{2}$ and $\rho$ is the density in $\mathrm{g} / \mathrm{cm}^{3} . \mu$ is the linear absorption coefficient in $\mu \mathrm{m}^{-1}$ and $\eta$ is the detector efficiency (52\% in the $\mathrm{C} 1 s$ region $^{18}$ ).

With a pixel size of $70 \mathrm{~nm}, 6 \mathrm{~ms}$ exposure time, and $1.25 \mathrm{~g} / \mathrm{cm}^{3}$ density of lipids we calculated the radiation dose per pixel applied during a 56 energy image sequence to be $\sim 1.2 \times 10^{7} \mathrm{~Gy}$. With a pixel size of $100 \mathrm{~nm}, 6 \mathrm{~ms}$ exposure time, and $1.055 \mathrm{~g} / \mathrm{cm}^{3}$ density of polystyrene particles the radiation dose applied during an image sequence (74 images) becomes $\sim 4.6 \times 10^{7} \mathrm{~Gy}$. In a study of polymethyl methacrylate, an organic polymer used as a photoresist, $50 \%$ mass loss was measured at a dose of $10^{7} \mathrm{~Gy} .{ }^{36}$ At least partial radiation damage of specimen during an acquisition must therefore be considered when taking image sequences (image stacks).

\section{NEXAFS spectra of phospholipid multilayers}

Phospholipids are important structural components of cell membranes. In its simplest form, a phospholipid is composed of one glycerol bonded to two fatty acids and one phosphate group. Phospholipids differ in terms of fatty acid chain length, degree of unsaturation (double bonds) in the fatty acids, and polar (phosphate-containing) groups present. Their fluidity decreases with chain length and increases according to the degree of unsaturation.

Dried lipid multilayers on $\mathrm{Si}_{3} \mathrm{~N}_{4}$ membranes were prepared as described in Sec. II. Figure 10 shows the image of a DMPC multilayer sample obtained by an optical imaging profiler. The thickness of multilayers in this sample was measured to be $\sim 120 \mathrm{~nm}$.

NEXAFS measurements were obtained by using a laboratory scale setup based on a laser-driven plasma source. The resolution of this spectrometer was experimentally determined to be $\lambda / \Delta \lambda \approx 200$ at $2.87 \mathrm{~nm}$. Nitrogen was used as target gas to calibrate the spectrometer. The spectrum of the nitrogen plasma, ignited under the same experimental condi- 


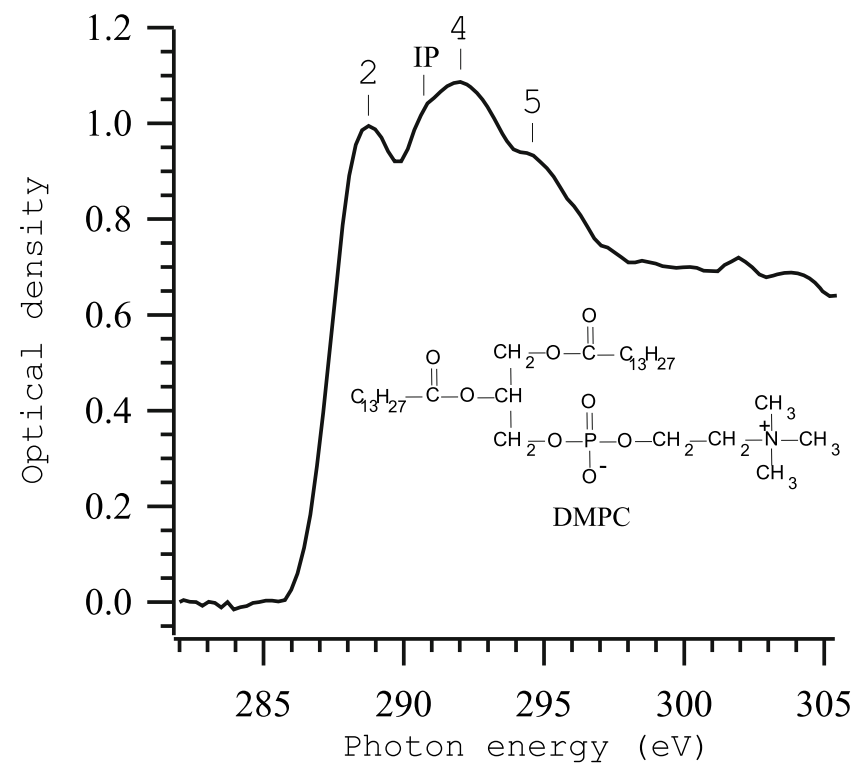

FIG. 11. NEXAFS spectra of the saturated DMPC phospholipid layer. Lines indicate locations of peaks and C $1 s$ IPs as estimated.

tions as krypton, consists of several isolated lines [e.g., $1 s^{2}-1 s 2 p$ transition of $\mathrm{N} \mathrm{VI}$ at $2.8787 \mathrm{~nm}$ (Ref. 37)] that were used to determine the dispersion relation of the grating. The accuracy of this calibration is better than $0.2 \mathrm{eV}$.

The emission spectra of the krypton plasma both with and without the sample were acquired, each obtained by an integration over 1000 pulses (total acquisition time of about 16 $\mathrm{min})$. The optical density was evaluated according to $\mathrm{OD}=$ $-\ln \left(I / I_{0}\right)$ and plotted as a function of energy.

The NEXAFS spectra of DMPC, DOPS, and DOPC lipid multilayers are displayed in Figs. 11 and 12. When approaching an edge by tuning the energy from the lower end to the edge, the $\pi^{*}$ resonances typically show first, followed by Rydberg and/or hydrogen-derived resonances, and above the ionization potential (IP), $\sigma^{*}$ resonances are present. The energies and proposed assignments are listed in Table I.

The spectrum of the saturated DMPC (Fig. 11) has three distinct spectral features: The $288.7 \mathrm{eV}$ peak might correspond to the $\pi^{*}(\mathrm{C}=\mathrm{O})$ resonance of the ester carbonyl group. A broad maximum at $292.2 \mathrm{eV}$ might be attributed to the $\sigma^{*}(\mathrm{C}-\mathrm{C})$ resonance. At $294.5 \mathrm{eV}$ the $\sigma^{*}$ feature can be assigned to the $\mathrm{C}-\mathrm{O}$ bond of the carboxylate group $\left(-\mathrm{CO}_{2}-\right)$.

Spectra of unsaturated DOPS and DOPC (Fig. 12) exhibit relatively strong, sharp peaks at 285.1 and $285 \mathrm{eV}$, respectively, assigned to the $\pi^{*}(\mathrm{C}=\mathrm{C})$ resonance. A shoulder at 288 $\mathrm{eV}$ in the spectrum of the DOPS and at $287.7 \mathrm{eV}$ in the spectrum of the DOPC might correspond to the $\pi^{*}(\mathrm{C}=\mathrm{O})$ resonance of the ester carbonyl group. The Rydberg/C $-\mathrm{H}^{*}$ resonances might be present at $289.4 \mathrm{eV}$ in the DOPC spectrum. The peak at $289.5 \mathrm{eV}$ in the DOPS spectrum is more pronounced and can be assigned to the overlap of the Rydberg/C $-\mathrm{H}^{*}$ resonances and $\mathrm{C} 1 s \rightarrow \sigma^{*}(\mathrm{C}-\mathrm{NH})$ transition in the $-\mathrm{CNH}_{3}^{+}$group of the serine. The broad maxima (features 4 and 5) in the continuum of both spectra are attributed to the $\sigma^{*}(\mathrm{C}-\mathrm{C})$ and $\sigma^{*}(\mathrm{C}-\mathrm{O})$ resonances, respec-

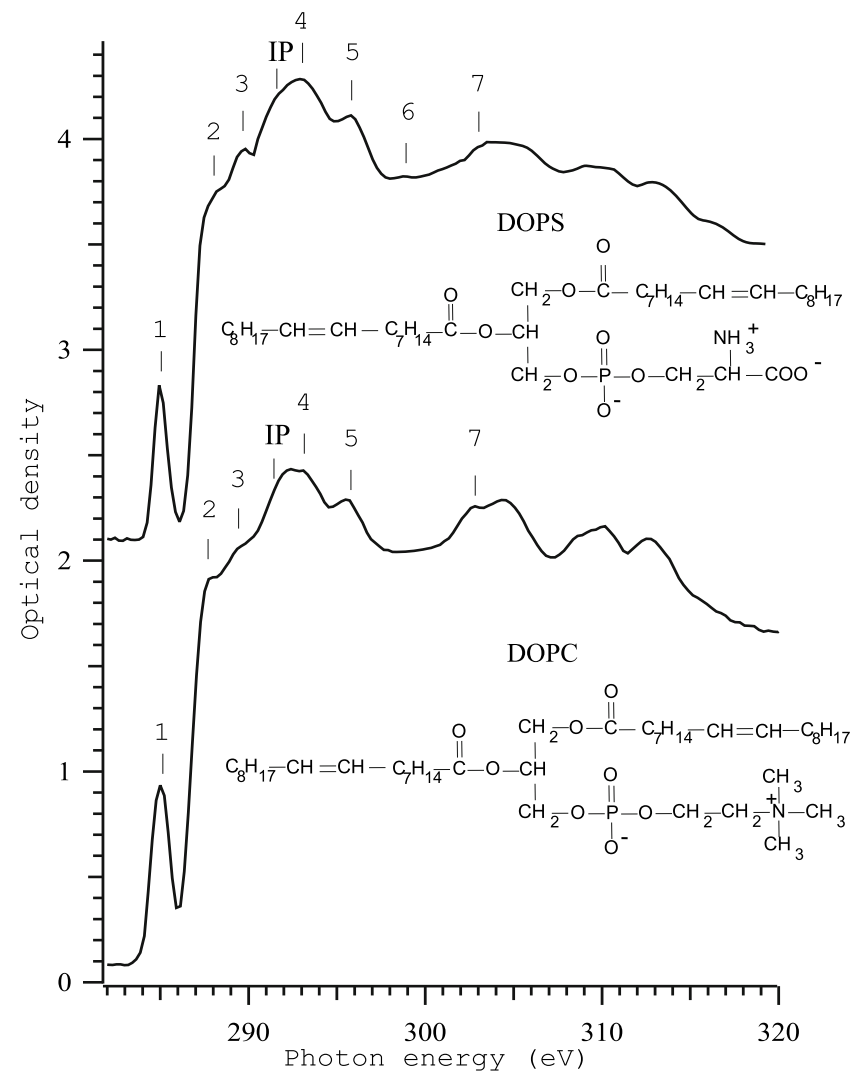

FIG. 12. NEXAFS spectra of unsaturated DOPS and DOPC phospholipids. Lines indicate locations of peaks and the IP as estimated. The spectra are shifted vertically for clarity.

tively. The $302.8 \mathrm{eV}$ peak in the DOPC spectrum and the $302.9 \mathrm{eV}$ peak in the DOPS spectrum might be the $\sigma^{*}(\mathrm{C}$ $=\mathrm{C})$ resonance. The DOPS spectrum contains the additional small peak at $298.7 \mathrm{eV}$ (feature 6), which is probably a $\sigma^{*}(\mathrm{C}=\mathrm{O})$ resonance in the carbonyl $\left(-\mathrm{COO}^{-}\right)$functional group. Features at $\sim 310 \mathrm{eV}$ cannot be attributed to the sample. We have checked that the features do not occur systematically in all spectra taken with this instrument by comparing our spectrum with spectra taken from other samples.

These assignments were made on the basis of inner-shell spectra for related compounds, such as complex alcohols, ${ }^{9}$ carboxylic acids, ${ }^{38}$ and amino acids. ${ }^{39}$

TABLE I. Energies and assignments of spectral features in the NEXAFS spectra of DMPC, DOPC, and DOPS.

\begin{tabular}{ccccc}
\hline \hline Feature & DMPC & $\begin{array}{c}\text { Energy }(\mathrm{eV}) \\
\text { DOPC }\end{array}$ & DOPS & Assignment \\
\hline 1 & & 285.1 & 285.0 & $1 \pi^{*}(\mathrm{C}=\mathrm{C})$ \\
2 & 288.7 & 287.7 & 288.0 & $\pi^{*}(\mathrm{C}=\mathrm{O})$ \\
3 & & 289.4 & 289.5 & Rydberg/C-H* \\
4 & 292.2 & 293.1 & 292.8 & $\sigma^{*}(\mathrm{C}-\mathrm{C})$ \\
5 & 294.5 & 295.6 & 295.8 & $\sigma^{*}(\mathrm{C}-\mathrm{O})$ \\
6 & & & 298.7 & $\sigma^{*}(\mathrm{C}=\mathrm{O})$ \\
7 & & 302.8 & 302.9 & $\sigma^{*}(\mathrm{C}=\mathrm{C})$ \\
& 290.8 & 291.7 & 291.5 & $\mathrm{IP}$ \\
\hline \hline
\end{tabular}




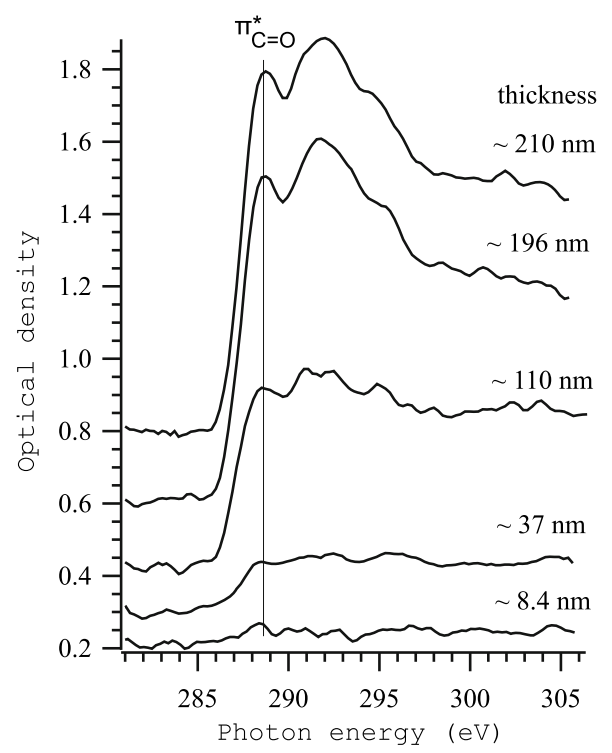

FIG. 13. Series of NEXAFS spectra of DMPC multilayers with different thicknesses. The $\pi^{*}(\mathrm{C}=\mathrm{O})$ resonance from the $\sim 37 \mathrm{~nm}$ thick layer can be still resolved.

We have tested the sensitivity of the NEXAFS measurement for the specific compact laboratory setup depending on the lipid film thickness. Figure 13 shows series of NEXAFS spectra in transmission of DMPC multilayers with different thicknesses. The thicknesses were estimated from optical densities. The $\pi^{*}(\mathrm{C}=\mathrm{O})$ resonance from $\sim 37 \mathrm{~nm}$ thick layer can be still resolved.

\section{SUMMARY AND DISCUSSION}

In summary, we have successfully used scanning transmission x-ray spectromicroscopy to image positively charged polystyrene latex microspheres binding to a supported twocomponent lipid membrane composed of charged and neutral lipid species. At the given parameters of vesicle fusion (i.e., in the absence of added calcium ions), the coverage was not complete, as expected. The presence of bilayer patches enabled us to investigate the sensitivity of scanning transmission x-ray spectromicroscopy down to the level of a thin lipid layer. However, current experimental conditions are at the limit of a sufficient signal-to-noise ratio, resulting in rather diffuse patch boundaries in the image. Nevertheless, preferential binding of microspheres to the oppositely charged bilayer was observed. Surprisingly, the bound micropheres aggregated on the lipid bilayer patch despite equal charges and interparticle repulsion. This is a clear demonstration of the rich and complex effects which can be observed in these multicomponent systems, where the degrees of freedom of the lipid composition as well as the lipid and colloid counterions has to be taken into account. For a clear-cut explanation, more data taken over an extended parameter range of ionic strength and surface charges need to be collected. While large microspheres as well as smaller fluorescence markers can be used in fluorescence microscopy to monitor the two-dimensional distribution of bound colloids at the lipid bilayer, the state of the lipid bilayer itself is more difficult to probe. This concerns, in particular, the degrees of freedom associated with demixing of charged and uncharged species on the relevant submicron length scales. Here synchrotron-based spectromicroscopy has the potential to chemically identify the individual species and to provide compositional maps. As the first step, we present a small data bank of the NEXAFS spectra of some of the most common lipids. Note that the spectroscopic data alone, without spatial resolution, can be collected on compact laser-driven plasma sources, as demonstrated here for the first time. Thereby, lipid NEXAFS spectroscopy is in principle amenable to a much larger number of laboratories, at least to the extent that such instrumentation becomes commercially available. However, it should be pointed out that high resolution data for organic molecules obtained with synchrotron sources contain valuable information, e.g., on vibrational fine structure. ${ }^{9}$ At the same time spectromicroscopy is likely to remain the realm of studies using synchrotron radiation. More strongly stated, even today, high resolution compositional maps of single-bilayer systems are at the technical limit but can be expected to benefit significantly from improvements in sources and beamline instrumentation. Therefore, the resolution and sensitivity limits of spectromicroscopy can be expected to shift in the future from a signal-to-noise related issue to an issue of dose-dependent radiation damage.

We have also presented NEXAFS results obtained by using a laboratory scale (compact) laser-driven plasma source. The carbon $K$-edge NEXAFS spectra of selected lipids, which differ in the headgroup structure or/and in containing the $\mathrm{C}=\mathrm{C}$ double bond in hydrocarbon tails, have been measured to provide a first step toward reference NEXAFS spectra of different lipids. As demonstrated here, soft X-ray NEXAFS can be carried out by compact instrumentation owing to the high peak flux, the broad emission spectrum, and the high dispersive power of the analyzing grating.

\section{ACKNOWLEDGMENTS}

The authors thank Ruth Szilluweit (Institut für Organische und Biomolekulare Chemie, Uni Göttingen) for help with AFM imaging and Frank Barkusky (Laser-Laboratorium Göttingen e.V.) for lipid multilayer imaging by optical imaging profiler. Financial support by SFB755 "Nanoscale Photonic Imaging" and European Network of Excellence on Soft Composite Materials (SOFTCOMP) is gratefully acknowledged.

${ }^{1}$ D. S. Dimitrov, Colloids Surf., A 282-283, 8 (2006).

${ }^{2}$ L. Ramos, T. C. Lubensky, N. Dan, P. Nelson, and D. A. Weitz, Science 286, 2325 (1999).

${ }^{3}$ S. Hong, J. A. Hessler, M. M. Banaszak Holl, P. Leroueil, A. Mecke, and

B. G. Orr, J. Chem. Health Safety 13, 16 (2006).

${ }^{4}$ S. May, J. Phys.: Condens. Matter 17, R833 (2005).

${ }^{5}$ M. Langner and K. Kubica, Chem. Phys. Lipids 101, 3 (1999).

${ }^{6}$ E. Sackmann, Science 271, 43 (1996).

${ }^{7}$ B. Maier and J. O. Rädler, Phys. Rev. Lett. 82, 1911 (1999).

${ }^{8}$ E. Nováková, K. Giewekemeyer, and T. Salditt, Phys. Rev. E 74, 051911 (2006).

${ }^{9}$ J. Stöhr, NEXAFS Spectroscopy (Springer-Verlag, New York, 1992).

${ }^{10}$ A. P. Hitchcock, C. Morin, Y. M. Heng, R. M. Cornelius, and J. L. Brash, 
J. Biomater. Sci., Polym. Ed. 13, 919 (2002).

${ }^{11}$ T. H. Watts, A. A. Brian, J. W. Kappler, P. Marrack, and H. M. McConnell, Proc. Natl. Acad. Sci. U.S.A. 81, 7564 (1984).

${ }^{12}$ R. Richter, A. Mukhopadhyay, and A. Brisson, Biophys. J. 85, 3035 (2003).

${ }^{13}$ S. G. Urquhart and H. J. Ade, J. Phys. Chem. B 106, 8531 (2002).

${ }^{14}$ G. Mitrea, J. Thieme, P. Guttmann, S. Heim, and S. Gleber, J. Synchrotron Radiat. 15, 26 (2008).

${ }^{15}$ C. Peth, F. Barkusky, and K. Mann, J. Phys. D 41, 105202 (2008),

${ }^{16}$ B. Niemann, G. Schneider, P. Guttmann, D. Rudolph, and G. Schmahl, in The New Göttingen X-Ray Microscope with Object. Holder in Air for Wet Specimens, X-Ray Microscopy IV, edited by V. V. Aristov and A. I. Erko (Bogorodskii Pechatnik, Moscow, 1994), p. 3035.

${ }^{17}$ U. Wiesemann, J. Thieme, P. Guttmann, B. Niemann, D. Rudolph, and G. Schmahl, AIP Conf. Proc. 507, 430 (2000).

${ }^{18} \mathrm{U}$. Wiesemann, "The scanning transmission $\mathrm{x}$-ray microscope at BESSY II,” Ph.D. thesis, Georg-August University Göttingen, 2003.

${ }^{19}$ S. Rehbein, J. Phys. IV 104, 207 (2003).

${ }^{20}$ L. Strüder et al., Nucl. Instrum. Methods Phys. Res. A 288, 227 (1990).

${ }^{21}$ B. L. Henke, E. M. Gullikson, and J. C. Davis, At. Data Nucl. Data Tables 54, 181 (1993)

${ }^{22}$ S. Kranzusch, C. Peth, and K. Mann, Rev. Sci. Instrum. 74, 969 (2003).

${ }^{23}$ J. Jass, T. Tjärnhage, and G. Puu, Biophys. J. 79, 3153 (2000).

${ }^{24}$ R. P. Richter, R. Brat, and A. R. Brisson, Langmuir 22, 3497 (2006).

${ }^{25}$ J. Raedler, H. Strey, and E. Sackmann, Langmuir 11, 4539 (1995).
${ }^{26}$ D. E. Leckband, C. A. Helm, and J. Israelachvili, Biochemistry 32, 127 (1993).

${ }^{27}$ M. C. Wiener, R. M. Suter, and J. F. Nagle, Biophys. J. 55, 315 (1989).

${ }^{28}$ M. Deserno and T. Bickel, Europhys. Lett. 62, 767 (2003).

${ }^{29}$ C. J. Buckley, Rev. Sci. Instrum. 66, 1318 (1995).

${ }^{30}$ U. Neuhäusler, S. Abend, C. Jacobsen, and G. Lagaly, Colloid Polym. Sci. 277, 719 (1999).

${ }^{31}$ C. Jacobsen, S. Williams, E. Anderson, M. T. Browne, C. J. Buckley, D. Kern, J. Kirz, M. Rivers, and X. Zhang, Opt. Commun. 86, 351 (1991).

${ }^{32}$ G. Gleber, J. Thieme, J. Niemeyer, and M. Feser, J. Phys. IV 104, 429 (2003).

${ }^{33}$ O. Dhez, H. Ade, and S. G. Urquhart, J. Electron Spectrosc. Relat. Phenom. 128, 85 (2003).

${ }^{34}$ A. P. Hitchcock, J. Synchrotron Radiat. 8, 66 (2001).

${ }^{35}$ T. Beetz and C. Jacobsen, J. Synchrotron Radiat. 10, 280 (2003).

${ }^{36}$ X. Zhang, C. Jacobsen, S. Lindaas, and S. Williams, J. Vac. Sci. Technol. B 13, 1477 (1995).

${ }^{37}$ NIST Atomic Spectra Database (http://physics.nist.gov/PhysRefData/ ASD/index.html).

${ }^{38}$ I. Ishii and A. P. Hitchcock, J. Electron Spectrosc. Relat. Phenom. 46, 55 (1988).

${ }^{39}$ K. Kaznacheyev, A. Osanna, C. Jacobsen, O. Plashkevych, O. Vahtras, H. Ågren, V. Carravetta, and A. P. Hitchcock, J. Phys. Chem. A 106, 3153 (2002). 